\title{
To Compare the Bacterial Reduction from the Infected Root Canals Using ProTaper Universal, WaveOne and NeoNiTi Instrumentation Systems
}

\author{
Renubala Sroa ${ }^{1}$, Baljeet Sidhu ${ }^{2}$, Neha Mengi ${ }^{2}$, Sarbjot Singh ${ }^{2}$, Maheep Sidhu ${ }^{3}$ * \\ ${ }^{1}$ Department of Conservative Dentistry and Endodontics, Punjab Government Dental College \& Hospital, Patiala, India \\ ${ }^{2}$ Department of Conservative Dentistry and Endodontics, Punjab Government Dental College \& Hospital, Amritsar, India \\ ${ }^{3}$ Department of Electronics \& Communications, Sri Ramaswamy Memorial University, Chennai, India
}

Email address:

drbaljeet@gmail.com (B. Sidhu),maheep98@outlook.com (M. Sidhu)

${ }^{*}$ Corresponding author

\section{To cite this article:}

Renubala Sroa, Baljeet Sidhu, Neha Mengi, Sarbjot Singh, Maheep Sidhu. To Compare the Bacterial Reduction from the Infected Root Canals Using ProTaper Universal, WaveOne and NeoNiTi Instrumentation Systems. International Journal of Dental Medicine.

Vol. 5, No. 2, 2019, pp. 40-45. doi: 10.11648/j.ijdm.20190502.11

Received: September 9, 2019; Accepted: October 9, 2019; Published: December 16, 2019

\begin{abstract}
Anticipation of root canal bacteria contamination is the foundation of endodontic treatment. The most predominant type is Enterococcus Faecalis strain, along with which several Gram negative enteric rods or Gram positive facultative may also be present. The aim of the present study was to compare the bacterial reduction from the infected root canals taking samples of pre-chemomechanical and post-chemomechanical preparation by ProTaper Universal, WaveOne and NeoNiTi instrumentation systems. Seventy-five systematically healthy patients was selected with permanent mandibular premolar teeth with single root and single canal. They are divided in three: Group I (ProTaper Universal), Group II (WaveOne) and Group III (NeoNiTi), in which each individual pre and post instrumentation sample of microorganism was taken and then analyzed using Paired ' $t$ ' test, One Way ANOVA with Post HOC comparison using Tukey test for microbiological evaluation. When pre-chemomechanical samples were compared by applying One-Way ANOVA test, the difference in prechemomechanical values in all three groups was statistically insignificant $(\mathrm{p}>0.05)$. Statistical analysis by applying paired ' $\mathrm{t}$ ' test, One-Way ANOVA and $p$ value showed that there was highly statistically significant change from pre-chemomechanical values to post-chemomechanical values in each study group $(p<0.001)$. There was highly statistically significant difference in CFU count percentage reduction between the pre-instrumentation and post-instrumentation values in all the groups $(p<0.001)$. Group I (ProTaper Universal) showed the highest percentage reduction followed by Group III (NeoNiTi) and Group II (WaveOne). Statistical analysis by paired ' $t$ ' test and $\mathrm{p}$ value showed that the reduction in number of colony forming units from S1 (pre-instrumentation) to S2 (post-instrumentation) was statistically highly significant $(\mathrm{p}<0.001)$. It may be concluded that the most effective instrumentation technique in eliminating microorganisms from the root canal was ProTaper Universal system in comparison to WaveOne and NeoNiTi.
\end{abstract}

Keywords: Protaper Universal, NeoNiTi, WaveOne, Paired ' $t$ ' Test, One-Way Anova and p Value

\section{Introduction}

Micro-organisms play a fundamental role in the aetiology of pulp and periapical diseases. They are an important factor affecting the result of endodontic treatment. Regardless of the number of sessions, an effective bacteriological control is mandatory. Amongst the diverse bacteria species found in endodontic infections, Enterococcus Faecalis stands out for its ability to grow in the presence or absence of oxygen, penetrate deep into dentinal tubules and survive adverse environmental conditions such as extreme alkaline $\mathrm{pH}$, high temperatures and scarce nutrition and association with persistent apical periodontitis, which makes it focus of research. [1]Reducing the bacterial count in infected root canals is accomplished by a combination of mechanical 
instrumentation, various irrigation solutions and antibacterial medicaments placed into the canal. [2] Although chemical agents are important in root canal instrumentation, some agents are not completely efficacious against all of the bacterial species in biofilms. However, mechanical instrumentation is the core method for bacterial reduction during endodontic treatment of infected root canals. During this stage, more than $90 \%$ of bacterial reduction is achieved. The mechanical instrumentation with a non-antimicrobial irrigant reduces the intracanal bacterial count enough to detect a quantifiable difference using appropriate sampling techniques. [3] Although manual instrumentation is commonly used by practitioners, automated rotary systems are associated with several advantages compared with manual techniques, including more rapid procedures, more centered preparations and less apical extrusion of debris, reduced stresses on both the operator and the patient and the number of files for instrumentation. [4] During the past decades, many systems have come out with single file systems [20] i.e OneShape (Micro Mega, Bescanson, France), WaveOne (DentsplyMaillefer, Ballaigues, Switzerland), and new NeoNiTi (Neolix, Creative Dental Instruments, Chảtres-la-Forẻt, France), which claims that a single file can be used for the entire preparation of root canals and it allows easier, faster and better root canal shaping. These instruments are designed with various tapers, greater than the 0.02 taper of traditional instruments, may results in excessive removal of dentin and high stress generation within the root canals and weakening of the root.

\section{Material and Method}

Seventy-five systemically healthy patients referred to the Department of Conservative Dentistry and Endodontics, Punjab Government Dental College \& Hospital, Amritsar for endodontic treatment were selected based on clinical and radiographic evaluation to compare the reduction in bacterialload in root canals by mechanical instrumentation using ProTaper Universal, WaveOne and NeoNiTi systems in permanent mandibular premolar teeth with single root and single canal. All volunteer patients signed an informed consent form. This research was approved by The Ethics committee of Punjab Government Dental College \& Hospital, Amritsar (Baba Farid University of Health Sciences, Faridkot).

All the selected teeth were single rooted with a primary endodontic infection showing the presence of 1 root canal and the absence of spontaneous pain periodontal pockets deeper than $4 \mathrm{~mm}$. None of the patients received antibiotic therapy within the preceding three months. Grossly decayed teeth that could not be isolated with a rubber dam were excluded. The following clinical/radiographic features were found in root canals with primary endodontic infections on investigation: negative response to pulp tests (electrical and cold), tenderness to percussion and a radiolucent area greater than $3 \mathrm{~mm}$ in size.

The operative field, including the tooth, clamp, and surroundings, were cleaned with $30 \%$ hydrogen peroxide (Fisher Scientific International Inc., Pittsburgh, United States) until no further bubbling of the peroxide occurs. All surfaces were then disinfected by vigorous swabbing with a $2.5 \% \mathrm{NaOCl}$ solution (Ricca Chemical Company, Arlington, TX, USA). The solutions were inactivated with $5 \%$ sodium thiosulfate to avoid interference with bacteriologic sampling.

Sterile round (SS-White New Jersey) and endo Z burs (Dentsply International, New York, U.S.) of suitable sizes were used to prepare the access cavity. After administering local anaesthesia, two stage access cavity was prepared without the use of water spray but under manual irrigation with sterile/apyrogenic saline solution and using a sterile high-speed bur. The first stage was performed to promote a major removal of contaminants, including carious lesions and restoration. In the second stage, before entering the pulp chamber, the access cavity was disinfected according to the protocol described above.

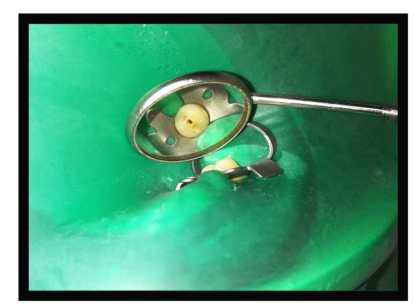

Figure 1. Prepared Access cavity under rubber dam isolation.

Pre-chemomechanical preparation sample (S1) was taken by placing two sterile paper points size 20 , into the canal, approximately $1 \mathrm{~mm}$ short of the root apex as estimated from the diagnostic radiographs for 60 seconds for sampling. Then with the help of sterile tweezer, first paper point was removed from the canal and immediately placed into vial containing thioglycollate broth for anaerobic bacteria and second paper point in peptone water broth for aerobic bacteria.

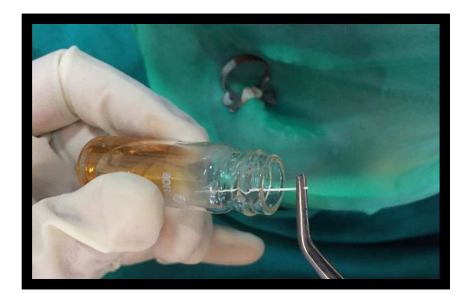

Figure 2. Sample transfer into peptone water for aerobic and anaerobic culture.

Finally samples were transferred to microbiological laboratory in respective broth for culturing. After accessing the pulp chamber and subsequent, pre-instrumentation microbial sampling, the pulp chamber was thoroughly cleaned with $2.5 \% \mathrm{NaOCl}$. Working length ( $1 \mathrm{~mm}$ short of the radiographic apex) was calculated with a radiograph after inserting \#10 K-file (Dentsply Maillefer, Ballaigues, Switzerland) in the canal and confirmed by Root ZX mini (J. Morita Corp., Kyoto, Japan) apex locator. According to the 
instrumentation technique used the selected teeth were randomly divided into three groups viz.: Group I (Protaper Universal), Group II (WaveOne) and Group III (NeoNiTi) comprising of twenty-five teeth each.

\section{GROUP I}

The teeth of selected patients in this group were prepared with ProTaper Universal instruments using a gentle in-andout motion. The canals were prepared in a crown-down fashion with the aid of CanalPro CL2 (Coltene, Whaledent, Switzerland) torque control endodontic electromotor. The instrumentation was done according to the manufacturer's instructions starting with SX and finishing with F4. The instruments (SX, S1, S2, F1, F2, F3 and F4) were used at a rotational speed of $300 \mathrm{rpm}$ with a torque of $3 \mathrm{~N}$. cm for SX \& S1, 1.5 N. cm for S2 \& F1, and 2 N. cm for F2, F3 and F4. The instrumentation sequence was: $\mathrm{SX}$ instrument at two thirds of the WL, S1 (taper $=0.02-0.11, \# 17)$, S2 (taper $=$ $0.04-0.115, \# 20), \mathrm{F} 1$ (taper $=0.055-0.07$, \#20), F2 (taper= $0.055-0.08, \# 25), \mathrm{F} 3$ (taper $=0.05-0.09, \# 30)$ and F4 (taper= $0.04-0.05, \# 40)$ at the working length. Canal patency was checked with a \#10 K-file (Dentsply Maillefer, Ballaigues, Switzerland) before the glide path, before using ProTaper S1, and after ProTaper S2 but before using the F1-F4 finishing files.

\section{GROUP II}

The selected teeth in this group were prepared by using WaveOne file system (DentsplyMaillefer, Ballaigues, Switzerland) file system. A \#40 WaveOne file with a 0.08 taper was used with the same endodontic electromotor operating at $350 \mathrm{rpm}$ with a $6: 1$ reduction hand piece in a reciprocating motion. The instrument was used in an in-andout pecking motion of about $3 \mathrm{~mm}$ in amplitude with apical pressure. After 3 pecking motions, the instrument was removed from the canal and cleaned. Next, \#15 K-type file was taken to the working length (WL) to check the canal patency. These procedures were repeated until the WaveOne instrument reached the WL.

GROUP III
The biomechanical preparation of selected teeth in this group was done by using NeoNiTi (Neolix, Creative Dental Instruments, Chảtres-la-Forẻt, France) file system at a rotational speed of $350 \mathrm{rpm}$ and $1.5 \mathrm{~N}$. cm torque with the aforementioned endodontic electromotor. First coronal third was prepared with NeoNiTiC1 files. After that the middle third of the canal was prepared with NeoNiTiA1 \#40 taper $4 \%$ using circumferential brushing action. A \#15 K-type file (DentsplyMaillefer, Ballaigues, Switzerland) was taken to the WL to check the canal patency. After that apical third was prepared with A1 using pecking motion.

In each group RC-Prep (Premier Dental, Norristown, PA, USA) was used as lubricant. Thorough irrigation with $2.5 \%$ $\mathrm{NaOCl}$ was carried out between consecutive files in each group using a $27 \mathrm{G}$ needle (Nipro Syringe, Maharashtra, India) in an up-and-down motion. Before the second sampling (S2) after instrumentation, $\mathrm{NaOCl}$ was inactivated with $5 \mathrm{ml}$ sterile $0.5 \%$ sodium thiosulfate during a 1-minute period, which was then removed with $5 \mathrm{ml}$ sterile/apyrogenic water.

Microbiological Culturing Procedure:

The microbiological culturing procedure was carried out in the Department of Microbiology, Government Medical College, Amritsar. Each screw capped vial was shaken to disperse the sample content evenly. In the laboratory, a gram stained smear was prepared from each specimen. The samples were directly inoculated with the help of inoculating loop onto MacConkey agar plates and incubated aerobically at $37^{\circ} \mathrm{C}$ for 24 hours. For anaerobes, specimens were directly inoculated on blood agar and incubated anaerobically in GasPak jar at $37^{\circ} \mathrm{C}$ for 48 hours. The plates were observed upto 7 days for any growth. After incubation period, the plates were examined and the colonies were counted with digital colony counter.

Statistical Analysis

The collected data were statistically evaluated using Paired 't' test, One Way ANOVA with Post HOC comparison using Tukey test for microbiological evaluation.

\section{Results}

Table 1. Mean reduction in pre-instrumentationand post-instrumentation values using paired 't'test in ProTaper Universal.

\begin{tabular}{llllll}
\hline \multirow{2}{*}{ Group } & S1 & & S2 & & Reduction from S1 to S2 \\
\cline { 2 - 6 } & Mean & SD & Mean & SD & Ralue \\
\hline Group I (ProTaper Universal) & $14.52 \times 10^{4}$ & $10.61 \times 10^{4}$ & $0.28 \times 10^{4}$ & $0.10 \times 10^{4}$ & $14.24 \times 10^{4} \pm 10.63$ \\
\hline
\end{tabular}

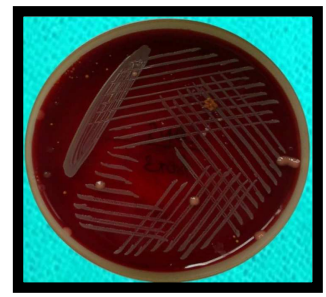

Figure 3. Aerobic culture plates showing colonies before chemomechanical preparation.

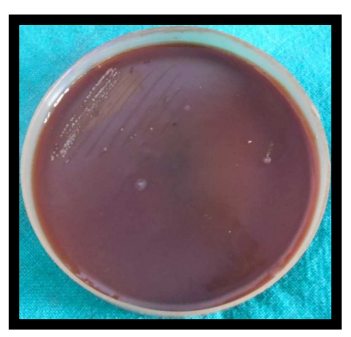

Figure 4. Aerobic culture plates showing colonies after chemomechanical preparation. 


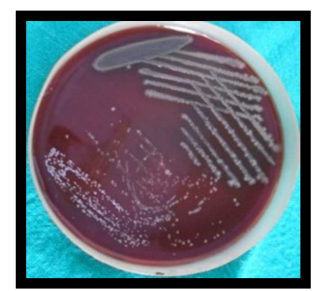

Figure 5. Anaerobic culture plates showing colonies before chemomechanical preparation.

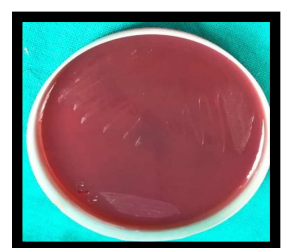

Figure 6. Anaerobic culture plates showing colonies after chemomechanicalpreparation.

Table 2. Mean reduction in pre-instrumentation and post-instrumentation values using paired 't' test in WaveOne.

\begin{tabular}{lllllll}
\hline \multirow{2}{*}{ Group } & S1 & \multicolumn{3}{l}{ S2 } & \multirow{2}{*}{ Reduction from S1 to S2 $_{\text {p value }}^{\#}$} \\
\cline { 2 - 6 } & Mean & SD & Mean & SD & & \\
\hline Group II (WaveOne) & $16.39 \times 10^{4}$ & $9.86 \times 10^{4}$ & $0.60 \times 10^{4}$ & $0.16 \times 10^{4}$ & $15.78 \times 10^{4} \pm 9.85$ & $<0.001^{* *}$ \\
\hline
\end{tabular}

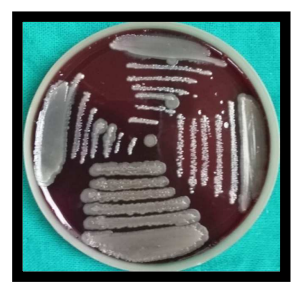

Figure 7. Aerobic culture plates showing colonies before chemomechanical preparation.

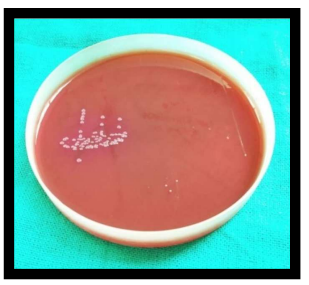

Figure 8. Aerobic culture plates showing colonies after chemomechanical preparation.

Table 3. Mean reduction in pre-instrumentation and post-instrumentation values using paired 't' test in NeoNiTi.

\begin{tabular}{lllllll}
\hline \multirow{2}{*}{ Group } & S1 & \multicolumn{3}{l}{ S2 } & \multirow{2}{*}{ Reduction from S1 to S2 $_{\text {p value }}^{\#}$} \\
\cline { 2 - 5 } & Mean & SD & Mean & SD & & \\
\hline Group III (NeoNiTi) & $17.47 \times 10^{4}$ & $9.49 \times 10^{4}$ & $0.43 \times 10^{4}$ & $0.17 \times 10^{4}$ & $17.03 \times 10^{4} \pm 9.54$ & $<0.001^{* *}$ \\
\hline
\end{tabular}

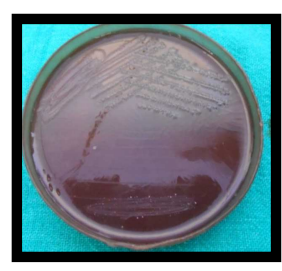

Figure 11. Aerobic culture plates showing colonies before chemomechanical preparation.

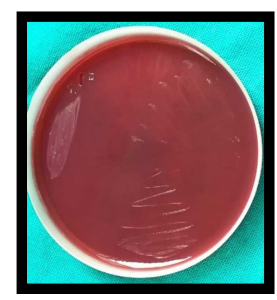

Figure 12. Aerobic culture plates showing colonies after chemomechanical preparation.
Figure 9. Anaerobic culture plates showing colonies before chemomechanical preparation.

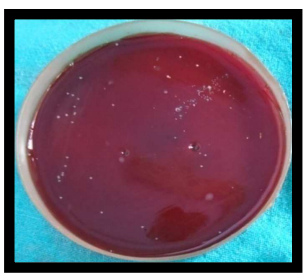

Figure 10. Anaerobic culture plates showing colonies after chemomechanicalpreparation.
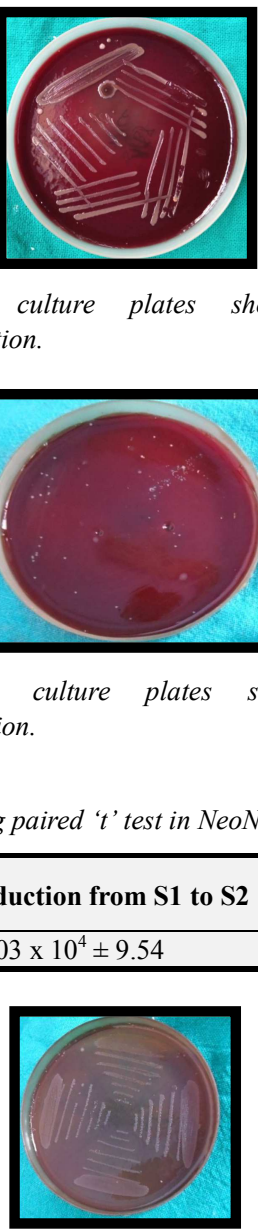

Figure 13. Anaerobic culture plates showing colonies before chemomechanical preparation.

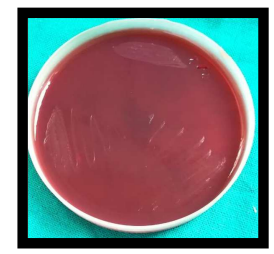

\#Paired ' $\mathrm{t}$ ' test; ${ }^{* *} \mathrm{p}<0.001$; Highly significant; SD- standard deviation0

Figure 14. Anaerobic culture plates showing colonies after chemomechanical preparation.

As per the table 4 , statistical analysis by paired ' $t$ ' test and $p$ 
value showed that the reduction in number ofcolony forming units from S1 (pre-instrumentation) to S2 (post-

instrumentation) was statistically highly significant $(\mathrm{p}<0.001)$.

Table 4. Meanandpercentage reduction in pre-instrumentation and post instrumentation values using one-way anova.

\begin{tabular}{|c|c|c|c|c|c|c|c|c|}
\hline \multirow{2}{*}{ Group } & \multicolumn{3}{|l|}{ S1 } & \multicolumn{3}{|l|}{ S2 } & \multirow{2}{*}{$\begin{array}{l}\text { \%age reduction } \\
\text { from } S 1 \text { to } S 2\end{array}$} & \multirow{2}{*}{ p value ${ }^{s}$} \\
\hline & Mean & SD & p value ${ }^{\$}$ & Mean & SD & p value ${ }^{\mathrm{s}}$ & & \\
\hline $\begin{array}{l}\text { Group I (ProTaper } \\
\text { Universal) }\end{array}$ & $14.52 \times 10^{4}$ & $10.61 \times 10^{4}$ & \multirow{3}{*}{$0.577^{\mathrm{NS}}$} & $0.28 \times 10^{4}$ & $0.10 \times 10^{4}$ & \multirow{3}{*}{$<0.001 * *$} & $96.82 \pm 2.63$ & \multirow{3}{*}{$<0.001^{* *}$} \\
\hline Group II (WaveOne) & $16.39 \times 10^{4}$ & $9.86 \times 10^{4}$ & & $0.60 \times 10^{4}$ & $0.16 \times 10^{4}$ & & $94.84 \pm 3.36$ & \\
\hline Group III (NeoNiTi) & $17.47 \times 10^{4}$ & $9.49 \times 10^{4}$ & & $0.43 \times 10^{4}$ & $0.17 \times 10^{4}$ & & $95.96 \pm 3.93$ & \\
\hline
\end{tabular}

\$One-Way ANOVA; NS: $\mathrm{p}>0.05 ; * * \mathrm{p}<0.001$; Highly significant

As per the table 5, statistical analysis by One-Way ANOVA and $p$ value showed statistically insignificant $(p>0.05)$ difference in pre- chemomechanical preparation values (S1) in the three groups indicating that the initial bacterial load was homogenous in all the tested groups. There was highly statistically significant difference in CFU count percentage reduction between the pre-instrumentation and postinstrumentation values in all the groups $(p<0.001)$. Group I (ProTaper Universal) showed the highest percentage reduction followed by Group III (NeoNiTi) and Group II (WaveOne).

Table 5. Inter group comparison using one-way anova along with Post-Hoc Tukey HSD.

\begin{tabular}{|c|c|c|c|c|c|c|}
\hline \multirow{2}{*}{ Group } & \multicolumn{2}{|l|}{ S1 } & \multicolumn{2}{|l|}{ S2 } & \multirow{2}{*}{$\%$ age reduction from $\mathrm{S} 1$ to $\mathrm{S} 2$} & \multirow{2}{*}{ p value ${ }^{\$}$} \\
\hline & Mean difference & p value ${ }^{\mathrm{s}}$ & Mean difference & p value ${ }^{\$}$ & & \\
\hline Group I vs. II $^{\#}$ & $1.86 \times 10^{4}$ & $0.788^{\mathrm{NS}}$ & $0.32 \times 10^{4}$ & $\mathrm{p}<0.001 * *$ & 1.98 & $0.098^{\mathrm{NS}}$ \\
\hline Group I vs. III ${ }^{\#}$ & $2.94 \times 10^{4}$ & $0.554^{\mathrm{NS}}$ & $0.15 \times 10^{4}$ & $\mathrm{p}<0.002 * *$ & 0.861 & $0.636^{\mathrm{NS}}$ \\
\hline Group II vs. III" & $1.08 \times 10^{4}$ & $0.923^{\mathrm{NS}}$ & $0.16 \times 10^{4}$ & $\mathrm{p}<0.001 *$ & 1.12 & $0.466^{\mathrm{NS}}$ \\
\hline
\end{tabular}

\#Post-Hoc Tukey HSD; NS: $\mathrm{p}>0.05$; Not significant; ${ }^{*} \mathrm{p}<0.05$; Significant; ${ }^{* *} \mathrm{p}<0.001$; Highly significant

As per the table 5, statistical analysis by One-Way ANOVA along with Post-Hoc Tukey HSD and $\mathrm{p}$ value showed no statistically significant difference $(\mathrm{p}>0.05)$ between Group I (ProTaper Universal), Group II (WaveOne) and Group III (NeoNiTi) instrumentation techniques. Thus, all the instrumentation techniques demonstrated similar effectiveness in reducing bacterial load.

\section{Discussion}

From the results above it is evident that all of the preparation techniques were effective at reducing the bacterial count [6]. The maximum number of CFU/ML reduction was found in root canals of teeth instrumented with ProTaper Universal, followed by NeoNiTi and WaveOne [7]. The better performance of ProTaper Universal may be attributed to the mechanical action of the instrumentation as in ProTaper Universal system multiple files were used compared to the single file systems (NeoNiTi and WaveOne). WaveOne and ProTaper Universal have a completely different design in their tip region. WaveOne single-file is characterized by three cutting edges with radial lands to support the blades and a relatively small chip space. Whereas, ProTaper have a triangular convex cross-section presenting no active cutting edges and a neutral rake angle which scraps the dentin surface. Moreover, ProTaper files have greater taper (F3 has 9\% taper and shaping files have progressively increasing tapers) as compared to WaveOne (8\% taper) and NeoNiTi (4\% taper). [8]

NeoNiTi showed superior efficiency in reducing bacterial population from root canals when compared to WaveOne [5]. This may be due to its EDM (Electrical Discharge Machining) manufacturing process which offers unique features such as progressive flexibility, sharp cutting edges and built in abrasive properties. The rough surface and abrasive properties may be responsible for better microbial reduction in root canal.

When pre-chemomechanical samples were compared by applying One-Way ANOVA and $p$ value, the difference in pre-chemomechanical values in all three groups was statistically insignificant $(\mathrm{p}>0.05)$ which means the prechemomechanical counts in all groups were in similar range. However, student paired ' $t$ ' test, One-Way ANOVA and $p$ value showed that there was highly statistically significant change from pre-chemomechanical values to postchemomechanical values in each study group $(p<0.001)$. It indicated that all the instrumentation systems tested in the study showed marked reduction in bacterial count after chemomechanical preparation [9].

From the discussion above, it may be inferred that WaveOne group showed lower level of bacterial load reduction when compared with ProTaper Universal and NeoNiTi rotary systems. However, no statistically significant difference ( $p>0.05$ ) was seen among the three tested groups. This implies that all the instrumentation systems used showed similar effectiveness in reducing cultivable bacteria from primarily infected root canal.

Therefore, ProTaper Universal, a multiple-file system, used in continuous rotation, having progressively tapered file design may be recommended as a potential instrumentation technique for biomechanical preparation of the root canals. However, before any definite conclusion can be drawn, clinical evaluation with larger number of samples and more extensive research with a definitive data distribution should be done to evaluate the three instrumentation techniques in future. 


\section{Conclusion}

The observation showed that the mean bacterial load $(\mathrm{CFU} / \mathrm{mL})$ found in the infected root canals before (S1) and after (S2) chemomechanical preparation in Group I (ProTaper Universal) was $14.52 \times 10^{4} \& 0.28 \times 10^{4}$, in Group II (WaveOne) was $16.39 \times 10^{4} \& 0.60 \times 10^{4}$ and in Group III (NeoNiTi) was $17.47 \times 10^{4}$ and $0.43 \times 10^{4}$. Thus, the percentage reduction in no. of CFU/mL from S1-S2 in Group I, II and III was $96.82 \%, 94.84 \%$ and $95.96 \%$ respectively [13]. The observations above showed that all instrumentation techniques appreciably reduced the bacterial count in the root canals [10]. When the bacterial reduction efficacy of Group I (ProTaper Universal), Group II (WaveOne) and Group III (NeoNiTi) was compared, the greatest percentage reduction was found in Group I followed by Group III and Group II. When pre-chemomechanical samples were compared by applying One-Way ANOVA test, the difference in prechemomechanical values [12] in all three groups was statistically insignificant $(\mathrm{p}>0.05)$ which means the prechemomechanical counts in all groups were in similar range [11]. Statistical analysis by applying paired ' $t$ ' test, One-Way ANOVA and $p$ value showed that there was highly statistically significant change from pre-chemomechanical values to post-chemomechanical values in each study group $(p<0.001)$. It indicated that all the instrumentation systems tested in the study showed marked reduction in bacterial count after chemomechanical preparation. By applying OneWay ANOVA followed by Post Hoc Comparison of Percentage Difference using Tukey HSD test, no statistical significant difference $(\mathrm{p}>0.05)$ was seen in all three groups. Under the conditions of present study, it may be concluded that the most effective instrumentation technique in eliminating microorganisms from the root canal was ProTaper Universal system in comparison to WaveOne and NeoNiTi. However, insignificant difference was seen among the three tested groups. Thus, ProTaper Universal, a multiplefile system, used in continuous rotation, having progressively tapered file design may be recommended as a potential instrumentation technique for biomechanical preparation of the root canal. However, before any definite conclusion can be drawn, clinical evaluation with larger number of samples and more extensive research with a definitive data distribution should be done to evaluate the three instrumentation techniques in future.

\section{References}

[1] Mehta, S, Singbal, KP, Merh, A \& Rai, R 2015, 'Evaluation of mechanical efficacy of Wave-One ${ }^{\mathrm{TM}}$ and F2 ProTaper ${ }^{\mathrm{TM}}$ using reciprocating motion and ProTaper ${ }^{\mathrm{TM}}$ system up to $\mathrm{F} 2$ using rotary motion in reducing E. faecalis count', $J$ Adv MedDent Scie Res, vol. 3, no. 1, pp. 19-25.
[2] Siqueira, JF, Lima, KC, Magalhaes, FAC, Lopes, HP \& Uzeda, M 1999, 'Mechanical Reduction of the Bacterial Population in the Root Canal by Three Instrumentation Techniques', J Endod, vol. 25, no. 5, pp. 332-335.

[3] Bystrom, A \& Sundqvist, G 1981, 'Bacteriologic evaluation of the efficacy of mechanical root canal instrumentation in endodontic therapy', Scand J Dent Res, vol. 89, pp. 321-328.

[4] Burklein, S, Benten, S \& Schafer, E 2014, 'Quantitative evaluation of apically extruded debris with different single-file systems: Reciproc, F360 and OneShape versus Mtwo', Int Endod J, vol. 47, pp. 405-409.

[5] Dalton, BC, Orstavik, D, Phillips, C, Pettiette, M \& Trope, M 1998, 'Bacterial Reduction with Nickel-Titanium Rotary Instrumentation', $J$ Endod, vol. 24, no. 11, pp. 763-767.

[6] Aydin, C, Tunca, YM, Senses, Z, Baysallar, M, Kayaoglu, G \& Orstavik, D 2007, 'Bacterial reduction by extensive versus conservative root canal instrumentation in vitro', Acta Odontol Scand, vol. 65, pp. 167-170.

[7] Baek, JY, Yoo, HM, Park, DS, Oh, TS, Kum, KY, Shin, SY \& Chang, SW 2014, 'Comparison of the shaping abilities of three nickel titanium instrumentation systems using microcomputed tomography', Journal of Dental Sciences, vol. 9, pp. 111-117.

[8] de Arruda Bitencourt, M, Rocha, DGP \& da Silveira Bueno, CE 2017, 'Incidence of Dentinal Defects on the External Apical Root Surface after Instrumentation with WaveOne Reciprocating Files at Different Working Lengths', J Endod, vol. 43, no. 3, pp. 491-495.

[9] Siqueira, JF, Alves, FRF, Almeida, BM, de Oliveira, JCM \& Rocas, IN 2010, 'Ability of Chemomechanical Preparation with Either Rotary Instruments or Self-adjusting File to Disinfect Oval-shaped Root Canals', J Endod, vol. 36, pp. 1860-1865.

[10] Neto, MM, Santos, SSF, Leao, MVP, Habitante, SM, Rodrigues, JRDD \& Jorge, AOC 2012, 'Effectiveness of three instrumentation systems to remove Enterococcus faecalis from root canals', IntEndod J, vol. 45, pp. 435-438.

[11] Nobrega, LMM, Gade-Neto, CR, Dametto, FR, Sarmento, CFM \&Carvalho, RA 2011, 'Ultrasonic irrigation in the removal of smear layer and Enterococcus faecalis from root canals', Braz J Oral Sci, vol. 10, pp. 221-225.

[12] Soares, JA, deCarvalho, MAR, Santos, SMC, Mendonc, RMC, Ribeiro-Sobrinho, AP, Brito-Junior, M, Magalhaes, PP, Santos, MH \& de Macedo Farias, L 2010, 'Effectiveness of Chemomechanical Preparation with Alternating Use of Sodium Hypochlorite and EDTA in Eliminating Intracanal Enterococcus faecalis Biofilm', J Endod, vol. 36, pp. 894 898.

[13] Villette, G, Manek, S, Legner, M, Fillery, ED, Torneck, CD, Basrani, BR \& Friedman, S 2008, 'Characterization of an Ex Vivo Model for the Assessment of Root Canal Disinfection', $J$ Endod, vol. 34, pp. 1490-1496. 